\title{
Suppression of SIRT2 and altered acetylation status of human pluripotent stem cells: possible link to metabolic switch during reprogramming
}

\author{
Ok-Seon Kwon ${ }^{1}$, Min-Joon $\mathrm{Han}^{2} \mathcal{E}$ Hyuk-Jin Cha ${ }^{1, *}$ \\ ${ }^{1}$ College of Natural Sciences, Department of Life Sciences, Sogang University, Seoul 04107, Korea, ${ }^{2}$ Department of Hematology, St. Jude \\ Children's Research Hospital, Memphis, Tennessee 38105, USA
}

\begin{abstract}
Primed human pluripotent stem cells (hPSCs) are highly dependent on glycolysis rather than oxidative phosphorylation, which is similar to the metabolic switch that occurs in cancer cells. However, the molecular mechanisms that underlie this metabolic reprogramming in hPSCs and its relevance to pluripotency remain unclear. Cha et al. (2017) recently revealed that downregulation of SIRT2 by miR-200c enhances acetylation of glycolytic enzymes and glycolysis, which in turn facilitates cellular reprogramming, suggesting that SIRT2 is a key enzyme linking the metabolic switch and pluripotency in hPSCs. [BMB Reports: Perspective 2017; 50(9): 435-436]
\end{abstract}

Most of the adenosine triphosphate (ATP) required to maintain cellular functions is produced in mitochondria via oxidative phosphorylation (OXPHOS). However, cancer cells display attenuated OXPHOS and consequently rely on ATP produced by glycolysis, even under aerobic conditions, through a process called the Warburg effect or aerobic glycolysis. Considering the prolonged hypoxic conditions that arise as cancer cell masses grow and the skewed dependency on glycolysis for ATP production, conversion of glucose to lactate via an altered metabolic pathway would be favorable for

*Corresponding author. E-mail: hjcha@sogang.ac.kr

https://doi.org/10.5483/BMBRep.2017.50.9.119

Received 5 July 2017

Keywords: Metabolism, miR-200c, Pluripotent stem cells, Reprogramming, SIRT2

Abbreviations: hESC, human embryonic stem cell; hPSC, human pluripotent stem cell; hDF, human dermal fibroblast; OXPHOS, oxidative phosphorylation

Perspective to: Cha Y, Han MJ, Cha HJ et al (2017) Metabolic control of primed human pluripotent stem cell fate and function by the iR-200c-SIRT2 axis. Nature Cell Biology 19, 445-456 (2017) doi: $10.1038 / \mathrm{ncb} 3517$ cancer cell survival, despite its low efficiency compared with that of OXPHOS. Interestingly, such a metabolic switch has been reported in human embryonic stem cells (hESCs), which share a variety of molecular properties with cancer cells (e.g., high proliferation capacity, high telomerase activity, and adaptation to hypoxic conditions), suggesting that common molecular events in cancer cells and hESCs underlies this metabolic reprogramming. Similarly, cells become highly dependent on glycolysis due to the reversion of cristae-poor mitochondrial structure during reprogramming with Yamanaka factors, which implies that this metabolic switch is important for the induction of pluripotency. However, the molecular mechanisms that govern aerobic glycolysis in human pluripotent stem cells (hPSCs) remain unclear.

Emerging evidence suggests that acetylation of proteins in the mitochondria, cytoplasm, and nucleus controls various cellular processes such as metabolism, implying that the acetylation status determines the metabolic switch in hPSCs. To investigate this, the acetyl proteome of hPSCs was compared with that of human dermal fibroblasts (hDFs) as a differentiated counterpart. This revealed that five glycolytic enzymes, namely, aldolase (encoded by ALDOA), glyceraldehyde-3-phosphate dehydrogenase (encoded by GAPDH), phosphoglycerate kinase (encoded by PGK1), enolase (encoded by ENO1), and pyruvate kinases (encoded by PKM1 and 2), are highly acetylated in hPSCs. The increased acetylation status of proteins is determined by either the high expression level of acetyltransferases, such as p300 and CBP, or low expression level of deacetylases, such as sirtuins, a group of multifunctional $\mathrm{NAD}^{+}$-dependent deacetylases that are highly evolutionarily conserved from bacteria to mammals. Meta-analysis using an open database followed by biochemical analysis revealed that SIRT2, one of seven sirtuins that mainly localizes in the cytoplasm, is clearly downregulated in hPSCs, while SIRT1, which predominantly localizes in the nucleus, is highly upregulated. Given that SIRT2 expression controls the acetylation status of the five aforementioned glycolytic enzymes, their enzymatic activities, and glucose metabolism, downregulation of SIRT2 is likely responsible for the metabolic switch observed in hPSCs. In particular, acetylation of lysine 322 of aldolase, a putative 
target of SIRT2, is responsible for its enzymatic activity, supporting the idea that SIRT2 is important for controlling glycolytic enzymes, aerobic glycolysis, and survival in hPSCs. In contrast with SIRT2, nuclear SIRT1 is upregulated in hPSCS and was recently reported to be important for maintaining hESC pluripotency by deacetylating p53. Consistently, depletion of SIRT2 in hDFs induces metabolic changes (e.g., a decreased oxygen consumption rate in mitochondria) that are favorable during cell reprogramming, suggesting that metabolic reprogramming precedes cell reprogramming. This led us to speculate that altered transcription during cellular reprogramming with Yamanaka factors suppresses SIRT2 and enhances aerobic glycolysis by increasing the acetylation of glycolytic enzymes. To elucidate the mechanism by which SIRT2 is suppressed, we first pooled a list of candidate miRNAs that target the 5'-untranslated region of SIRT2 and whose expression is regulated by Yamanaka factors and is highly associated with cellular reprogramming. Among four miRNAs (miR-25, -92b, -200c, and -367) that are hPSCspecific, miR-200c is responsible for SIRT2 downregulation in hPSCs. It is noteworthy that miR-200c expression is regulated by OCT4 and induces mesenchymal-to-epithelial transition, which is a critical event in cell reprogramming. Furthermore, miR-200c is one of the miRNAs that were used to induce reprogramming without Yamanaka factors in the independent study.

Collectively, expression of OCT4 during cell reprogramming induces miR-200c, which leads to suppression of SIRT2. Downregulation of SIRT2 enhances acetylation status of glycolytic enzymes such as aldolase, GAPDH, enolase, and PGK1, and leads to an increase in aerobic glycolysis, which may be an important event preceding cell reprogramming (Diagram). However, it is important to note that mouse embryonic stem cells (mESCs) are bivalent in their energy production (e.g., switch from glycolysis to OXPHOS on demand), similar to other somatic cells, and undergo metabolic switch, once committed to differentiate into epiblast stem cells (EpiSCs). There is emerging evidence that hPSCs differing from mESCs in terms of their embryonic status (e.g., naïve vs. primed), resembles the EpiSCs, which accounts for a variety of their specific cellular and molecular properties of hPSCs compared to those of mESCs, including aerobic glycolysis. A switch from bivalent energy metabolism to

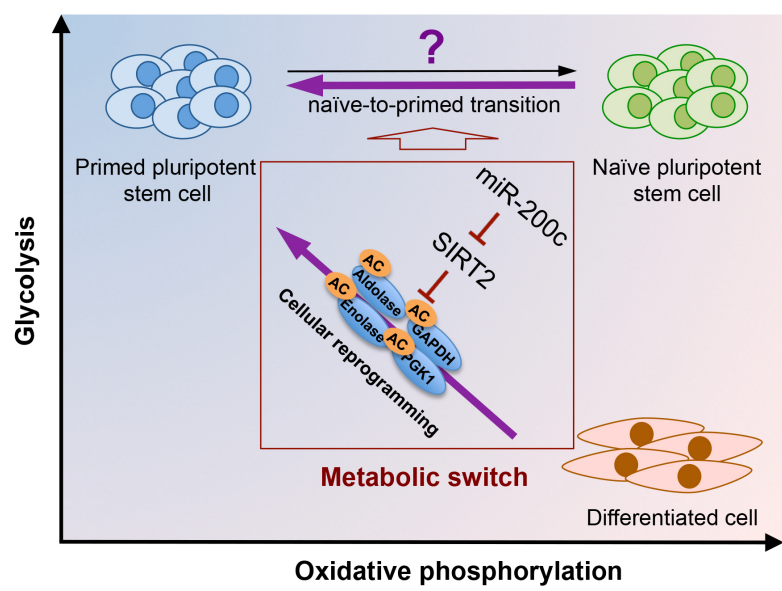

Diagram. Proposed model for metabolic switch by miR-200cSIRT2 in hPSCs During cellular reprogramming for hPSCs, which is 'primed state', downregulation of SIRT2 expression by miR-200c increased acetylation status of glycolytic enzymes, leading to the metabolic switch from OXPHOS to glycolysis. Roles of altered acetylation in bivalent metabolic status of naïve hPSCs should be further characterized in future. ' $A C^{\prime}$ indicates increased acetylation.

aerobic glycolysis occurs when naïve hESCs, which is similar to that of mESCs, are converted from a "primed" state of pluripotency (similar to mouse EpiSCs). Such "metabolic exit" results from increased tri-methylation of histone 3 lysine 27 (H3K27me3), which in turn induces the hypoxia-inducing factor $1 \alpha$ (HIF1a) pathway for the naïve-to-primed transition. While multiple protocols are being established to convert hPSCs into a naïve state, it would be interesting to examine the role(s) of the acetylation status, determined by sirtuins, especially SIRT2, in naïve pluripotency. Importantly, SIRT1, which is highly expressed in hPSCs, was recently revealed to govern naïve pluripotency by deacetylating OCT4.

\section{ACKNOWLEDGEMENTS}

This work was supported by the National Research Foundation of Korea (NRF) grant funded by the Korea government (MSIP) (No. 2009-0093822, 2011-0030043, and 2017R1A2A2A0500 0766). 